\title{
Miranda
}

Revue pluridisciplinaire du monde anglophone /

Multidisciplinary peer-reviewed journal on the English-

speaking world

$14 \mid 2017$

Early American Surrealisms, 1920-1940 / Parable Art

\section{John Gay, Trivia et autres vues urbaines}

\section{Xavier Cervantès}

\section{OpenEdition}

\section{Journals}

Édition électronique

URL : http://journals.openedition.org/miranda/10011

DOI : 10.4000/miranda.10011

ISSN : 2108-6559

Éditeur

Université Toulouse - Jean Jaurès

Référence électronique

Xavier Cervantès, « John Gay, Trivia et autres vues urbaines », Miranda [En ligne], 14 | 2017, mis en ligne le 03 avril 2017, consulté le 16 février 2021. URL : http://journals.openedition.org/miranda/10011 ; DOI : https://doi.org/10.4000/miranda.10011

Ce document a été généré automatiquement le 16 février 2021.

\section{c) (i)}

Miranda is licensed under a Creative Commons Attribution-NonCommercial-NoDerivatives 4.0 International License. 


\title{
John Gay, Trivia et autres vues urbaines
}

\author{
Xavier Cervantès
}

\section{RÉFÉRENCE}

Gay, John, Trivia et autres vues urbaines, trad. et éd. Jacques Carré, Paris, Classiques Garnier (collection « Littératures du monde »), 2016, 354 pages, ISBN :

978-2-8124-4576-7

1 Jacques Carré propose dans cet ouvrage une traduction (avec texte original en regard) de divers opuscules poétiques (ainsi que de la «farce tragi-comique» The Mohocks) écrits par John Gay dans sa première phase de création, entre 1712 et 1725, soit plus ou moins longtemps avant l'œuvre à laquelle il doit l'essentiel de sa gloire littéraire aujourd'hui, The Beggar's Opera (1728). Cette traduction, annotée et assortie d'une copieuse introduction de près de cinquante pages ainsi que d'une bibliographie et d'un index, est la toute première dans notre langue, ce qui permet d'approfondir notre connaissance d'un auteur dont la production est trop souvent réduite essentiellement à un unique chef-d'œuvre occultant ses autres opus. Elle ne hisse certes pas Gay au même rang que les deux grandes figures de la poésie du premier $18^{\text {ème }}$ siècle que sont Pope et Swift, mais rappelle utilement son importance au Parnasse de l'époque dite augustéenne.

2 Le fil rouge qui parcourt cette anthologie est, comme son titre le suggère, le portrait que brosse Gay de Londres, métropole tentaculaire et chaotique au sein de laquelle le brassage social est de rigueur, où le marquis côtoie le vide-gousset et la fille de joie, où la fange et les odeurs nauséabondes sont omniprésentes, tout autant que le risque d'une rencontre malheureuse à un carrefour (sous l'égide de la déesse romaine Trivia, qui donne son nom à la pièce maîtresse de l'anthologie). Cette thématique permet de réunir ces instantanés de la vie métropolitaine que sont Trivia : or, The Art of Walking the 
Streets of London, quatre "town eclogues ", la farce mentionnée précédemment, deux épîtres et deux autres opuscules mineurs.

L'introduction, dense quoique d'une lecture toujours aisée, est un modèle du genre. Le traducteur y présente et contextualise chacune des pièces sélectionnées et, surtout, en s'appuyant sur des études récentes ou plus anciennes, propose plusieurs pistes exégétiques possibles et cumulables entre elles. Ces clefs de lecture variées mettent bien en exergue la richesse et la polyvalence des textes de Gay, aussi légers, anecdotiques voire ... triviaux qu'ils puissent paraître parfois. Au-delà de la simple et évidente satire de la société et des mœurs de son temps (principalement satire de la consommation effrénée, du luxe et de la décadence morale de la nation et en particulier de son élite sociale), il apparaît clairement que d'autres clefs de lecture peuvent être utilisées pour mettre au jour toutes les facettes des textes réunis. C'est ainsi que la piste biographique n'est pas négligée (les rapports ambivalents et parfois houleux de l'écrivain avec l'univers du mécénat littéraire et celui des imprimeurs et du marché de l'édition, en particulier), de même que sont proposés divers éléments de contextualisation sociale et économique très éclairants.

4 Est aussi proposée la piste génétique : les différents textes du recueil contiennent en effet de nombreux jeux référentiels avec la tradition latine (Horace, Ovide, Virgile ou Juvénal); est ainsi décliné tout un vaste éventail de possibilités, de l'emprunt ou de la citation à l'adaptation en vue de la création d'un genre poétique nouveau (les « town eclogues »), en passant par l'imitation ou encore le pastiche. Le dialogue avec le canon classique et ses grands genres poétiques (épître, épopée et surtout pastorale), via les modernes (Milton, Dryden, Swift ou Pope), s'avère particulièrement fécond et confère une épaisseur intertextuelle parfois inattendue aux textes sélectionnés.

Les possibilités interprétatives sont donc multiples et le traducteur a la modestie de les évoquer toutes sans en privilégier ni en imposer aucune en particulier (il en va de même en ce qui concerne les intentions et convictions de l'auteur, souvent ambivalentes, voire contradictoires). En replaçant l'écriture de Gay dans le contexte de l'esthétique rococo alors naissante et en rapprochant ses tableautins de la vie londonienne des peintures ou gravures de Hogarth ou de Marcellus Laroon, ce dernier instaure par ailleurs un dialogue intersémiotique très évocateur entre littérature et culture visuelle.

6 La traduction elle-même est satisfaisante et fluide. Loin des « belles infidèles » du $18^{\text {ème }}$ siècle, elle est précise et scrupuleuse, tout en restant alerte. Jacques Carré semble avoir apporté une attention particulière à bien rendre, au sein de chaque pièce, le mélange parfois surprenant de plusieurs tonalités disparates, du sérieux au burlesque en passant par l'héroï-comique. L'équilibre entre l'imitation du style d'époque et des touches résolument plus moderne est soigneusement maintenu. 
INDEX

Keywords : 18th-century poetry, London, satire, neo-classicism, literary patronage

Mots-clés : poésie du 18ème siècle, Londres, satire, néo-classicisme, mécénat littéraire

\section{AUTEURS}

\section{XAVIER CERVANTÈS}

Professeur des Universités

Université Toulouse Jean Jaurès

xavier.cervantes@wanadoo.fr 